\title{
Disseminated Mycobacterium avium disease with nodular infiltration in the small intestine, detected by capsule endoscopy
}

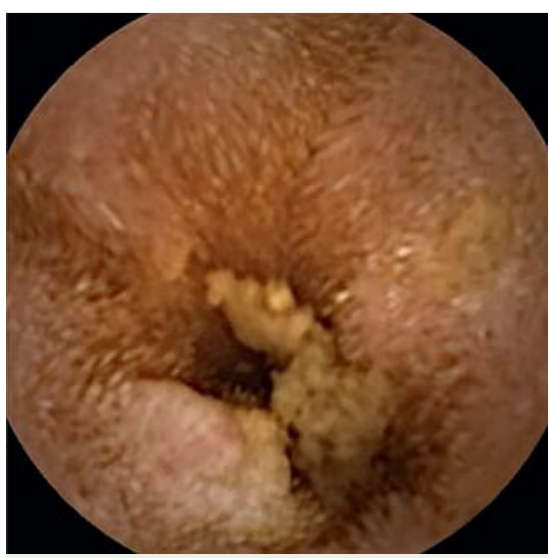

Fig. 1 Capsule endoscopy revealed a whitish nodular pattern almost throughout the small intestine.
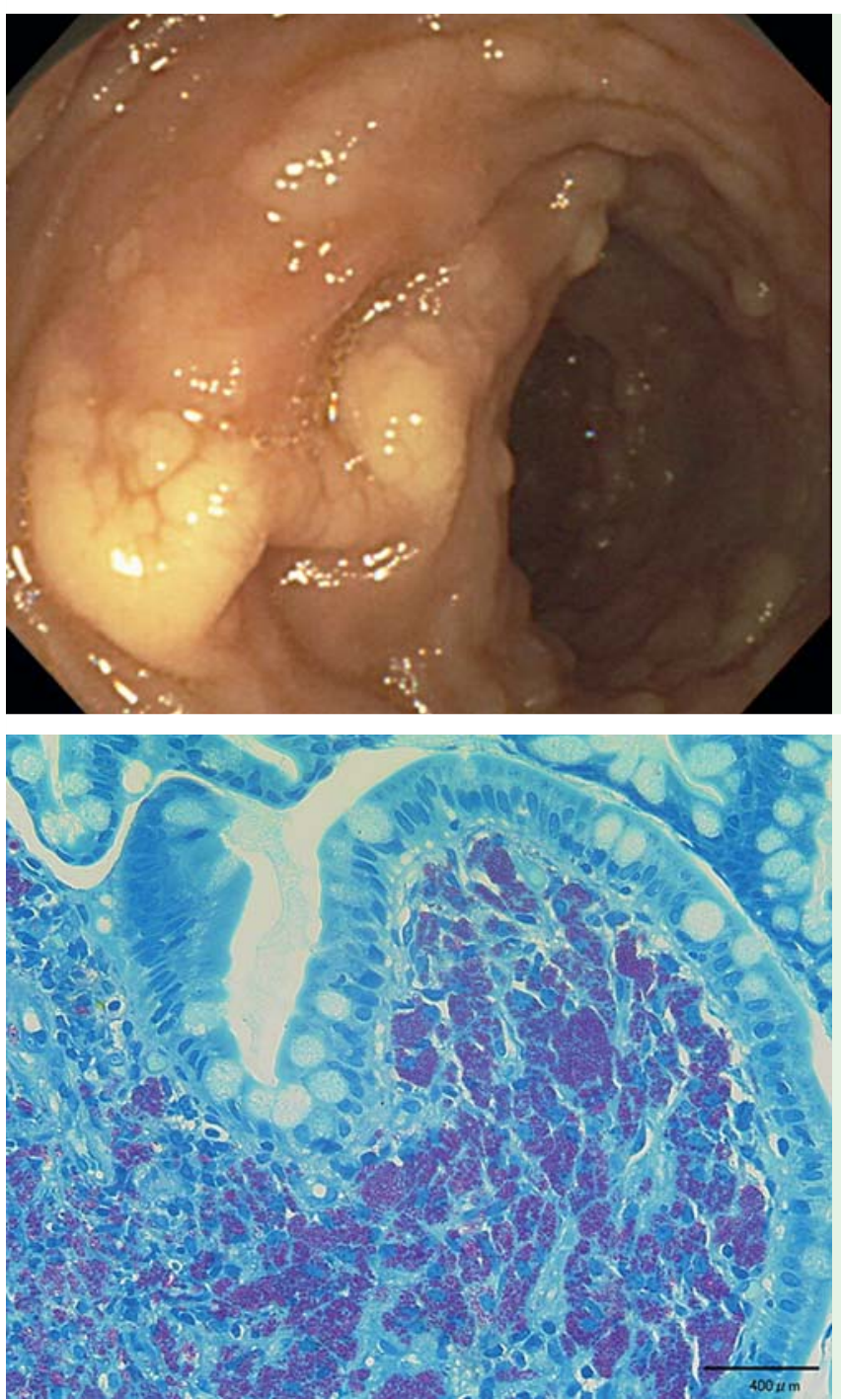

A 42-year-old man with human immunodeficiency virus (HIV) infection presented with abdominal pain and diarrhea, and was admitted to our hospital. His CD4 count was 10 cells $/ \mathrm{mm}^{3}$, and he was under highly active antiretroviral therapy (HAART). A chest radiograph revealed cavitary disease, and sputum examination by polymerase chain reaction (PCR) was positive for Mycobacterium avium. We performed gastroscopy and colonoscopy to identify the cause of the abdominal pain and diarrhea, but the examinations revealed no abnormalities, and a fecal culture was negative. Therefore, a capsule endoscopy was performed, which

Fig. 2 Enteroscopic view showing scattered white nodules and plaques in the ileum.

Fig. 3 Histological view of ileum biopsy tissue after staining with Ziehl-Neelsen stain; original magnification $\times 400$. revealed multiple white nodules almost throughout the small intestine ( $\bullet$ Fig. 1). Therefore, enteroscopy was performed for detailed examination and biopsy (๑ Fig. 2). Biopsy showed granuloma formation and numerous macrophages containing abundant Ziehl-Neelsen stain-positive bacilli ( $\bullet$ Fig. 3). Fecal culture was performed again to identify the bacteri$\mathrm{um}$, and the result was positive for Mycobacterium avium. Therefore, a diagnosis of gastrointestinal infection caused by Mycobacterium avium was made in this patient with advanced acquired immunodeficiency syndrome (AIDS). The lesions in the small intestine were thought to be secondary lesions - a metastatic infection from the lung. The symptoms gradually improved with continuation of treatment directed against Mycobacterium avium complex (MAC).

Mycobacterium avium is the most common mycobacterium implicated in infections of the gastrointestinal tract in patients with AIDS. The most common site of involvement is the duodenum, and involvement of the jejunum and ileum is rarely found [1]. One of the reasons why diagnosis was delayed was that besides small-intestinal lesions being rare, no minimally invasive modality to ascertain lesions of the small intestine was available until capsule endoscopy was developed. Capsule endoscopy allows examination of the small intestine without causing strain, and was very useful in this case. Use of capsule endoscopy may allow more small-intestinal lesions to be recognized in patients with AIDS.

\section{Endoscopy_UCTN_Code_CCL_1AC_2AH}

\section{Competing interests: None}

\author{
E. Yamada ${ }^{1}$, H. Endo ${ }^{1}$, A. Nakajima ${ }^{1}$, \\ S. Yamanaka² \\ Division of Gastroenterology, Yokohama \\ City University School of Medicine, \\ Yokohama, Japan \\ Department of Pathology, \\ Yokohama City University School of \\ Medicine, Yokohama, Japan
}




\section{References}

1 Sun HY, Chen MY, Wu MS et al. Endoscopic appearance of GI mycobacteriosis caused by the Mycobacterium avium complex in a patient with AIDS: case report and review. Gastrointest Endosc 2005; 61: 775 - 779
Bibliography

DOI $10.1055 / \mathrm{s}-0030-1256782$

Endoscopy 2011; 43: E332-E333

(c) Georg Thieme Verlag KG Stuttgart · New York . ISSN 0013-726X
Corresponding author

\section{H. Endo, MD}

Division of Gastroenterology

Yokohama City University School of Medicine

3-9 Fukuura

Kanazawa-ku

Yokohama 236-0004

Japan

Fax: +81-45-7843546

endo1978@yokohama-cu.ac.jp 